\title{
Kinematical analysis of Bipolar Planetary Nebulae
}

\author{
Martina Dobrinčić ${ }^{1}$, Eva Villaver ${ }^{2}$, Martín A. Guerrero ${ }^{3}$ \\ \& Arturo Manchado ${ }^{1}$ \\ ${ }^{1}$ Instituto de Astrofísica de Canarias, 38200 La Laguna, Tenerife, Canary Islands, Spain \\ ${ }^{2}$ Space Telescope Science Institute, 3700 San Martin Drive, Baltimore MD 21218, USA; \\ Affiliated to the Hubble Space Telescope Department of ESA \\ ${ }^{3}$ Instituto de Astrofísica de Andalucía, Granada, Spain
}

\begin{abstract}
Bipolar planetary nebulae (BPNe) offer a unique opportunity to test models that aim to reproduce the PNe morphologies. In particular, kinematic studies of BPNe allow a reconstruction of the 3D structure of the nebula, otherwise hidden in imaging studies. With this aim in mind we have obtained long-slit echelle spectra of a sample of PNe which cover the full range of observed bipolar morphologies, from elliptical to highly collimated. The analysis of our kinematical data reveals equatorial expansion velocities in the low to medium range (3 to $16 \mathrm{~km} \mathrm{~s}^{-1}$ ), while the polar expansion velocities range from 18 to $100 \mathrm{~km} \mathrm{~s}^{-1}$. We find that the kinematics of the PN K 3-46 can only be explained by a decrease in the expansion velocity with time. The kinematical ages, calculated by using distances estimated from Galactic rotation curves, when available, or by using statistical values, show that the BPNe in our sample - even those which show non-extreme collimation - appear to be young. We have compared our results with the latest theoretical models of BPN formation, and find good agreement between the observed expansion velocities and the numerical models that use magnetic fields coupled with stellar rotation as the collimation mechanism.
\end{abstract}

Keywords. planetary nebulae: kinematics and dynamics

\section{Analysis and Results}

The long-slit echelle spectra were obtained at the 4.2-m William Herschel Telescope using the Utrecht Echelle Spectrograph with a spectral resolution of $6.5 \mathrm{~km} \mathrm{~s}^{-1}$. The spectra were reduced using the standard IRAF tasks for two-dimensional spectra and the kinematical analysis was performed using the empirical kinematical model of Solf \& Ulrich (1985).

Listed in Table 1 are: in column (1), the most common PNe name; in column (2) and (3), the best values of the polar $\left(\mathrm{V}_{p}\right)$ and equatorial $\left(\mathrm{V}_{e}\right)$ expansion velocities respectively obtained from our fits to the spectra; column (4) gives the kinematical age at $1 \mathrm{kpc}$; column (5) the real age using the distances given in column (6), column (7) the derived inclination from the spectra; and column (8), the $\gamma$-factor derived from our fits to Solf's model (increasing value with increasing elongation in the polar direction). As Solf's model does not handle well highly collimated objects such us Hen 2-437, a range of possible values is given for this object. Two sets of values are given for M 1-75 as it is quadrupolar.

The kinematical ages given in column (4) of Table 1 were obtained assuming uniform nebular expansion and assuming that all the nebula are located at $1 \mathrm{kpc}$. To estimate the PN age in column (5), we have used combined distance values taken from the rotation curves of Burton (1974) and statistical distances given in the Acker et al. (1992) catalogue. The objects that show pronounced collimated structures, i.e large polar to equatorial 
Table 1. Results

\begin{tabular}{lccccccc}
\hline Object & Vp & $\begin{array}{r}\mathrm{Ve} \\
\mathrm{km} \mathrm{s}^{-1}\end{array}$ & $\begin{array}{c}\text { K.Age } \\
\mathrm{yr}\end{array}$ & Age & $\begin{array}{c}\text { Dist. } \\
\mathrm{Kpc}\end{array}$ & $\begin{array}{c}\text { inclination } \\
\text { degrees }\end{array}$ & $\gamma$ \\
\hline Hen 2-428 & 80 & 16 & 2400 & 5280 & 2.2 & -75 & 1 \\
M 4-14 & 65 & 11 & 1500 & 5550 & 3.7 & 38 & 5 \\
K 3-58 & 39 & 12 & 1800 & 11070 & 6.2 & -57 & 2.2 \\
M 2-48 & 100 & 10 & 1160 & 4830 & 4.2 & -79 & 8 \\
Hen 2-437 & $50 . .100$ & $(5)$ & $750 . .2000$ & & & -89 & 20 \\
M 1-75 & 55 & 8 & 2700 & 9100 & 3.4 & 87 & 6 \\
& 45 & 12 & 2400 & 9100 & 3.4 & 65 & 5.5 \\
WeSb 4 & 95 & 14 & 3400 & 15980 & 4.7 & 50 & 6 \\
K 3-46 & 18 & 3 & 11000 & 24300 & 2.7 & -70 & 0.9 \\
M 3-55 & 19.5 & 6 & 1800 & 5040 & 2.8 & 40 & 0.6 \\
\hline
\end{tabular}

ratios, are expected to be young. Among these PNe are He 2-428, M 4-14, M 2-48, and M 3-55. PNe with deteriorated shapes such as M 1-75 and WeSb4 are expected to be older. The ages calculated in Table 1 fit perfectly well into this picture with two exceptions: K 3-46, which very likely has suffered a decrease of the expansion velocity with time, and $\mathrm{K} 3-58$, which is the most distant object in our sample. Our kinematical fitting does not apply to K 3-46 since the model assumes uniform expansion, thus the actual age of this object could be much lower than the high value we have found.

From a modelling view point, PNe shaping requires combining the basic idea of interactive stellar winds of Kwok et al. (1978) with a collimation mechanism. Numerical models that include stellar rotation together with a magnetized wind have been performed by García-Segura et al. (1999). Moreover some observational evidence for the presence of magnetic fields has recently been provided by Jordan et al. (2005) in the central stars of a handful of PNe. In order to test the viability of magnetohydrodynamical models as the main collimation mechanism in $\mathrm{PNe}$, we have compared our kinematic results with the numerical models of García-Segura et al. (1999) and García-Segura \& López (2000). As an example, the objects in our sample that present the lowest $\gamma$ factors (M 3-55, K 3-46 and Hen 2-428) also show similar ratios of polar to equatorial expansion velocities and compare very well with the models from García-Segura et al. (1999) that use rapid stellar rotators. In general, the kinematics and the morphology of all of our objects fit very well with all the García-Segura \& López (2000) models.

\section{References}

Acker, A., Marcout, J., Ochsenbein, F., Stenholm, B., \& Tylenda, R. 1992, Garching: European Southern Observatory, 1992

Burton W.B. 1974 "The large scale distribution of neutral hydrogen" in Galactic and Extragalactic Radio Astronomy eds. G. Verschuur \& K.I. Kelleman (Springer Verlag)

García-Segura, G., Langer, N., Różyczka, M., \& Franco, J. 1999, ApJ 517, 767

García-Segura, G., \& López, J. A. 2000, ApJ 544, 336

Jordan, S., Werner, K., \& O'Toole, S. J. 2005, A\& A 432, 273

Kwok, S., Purton, C. R., \& Fitzgerald, P. M. 1978, ApJL, 219, L125

Solf, J., \& Ulrich, H. 1985, A\&A 148, 274 